\title{
ERRATUM
}

\section{Binary fluorous tagging enables the synthesis and separation of a 16-stereoisomer library of macrosphelides}

Dennis P. Curran, Mantosh K. Sinha, Kai Zhang, Jesse J. Sabatini and Dae-Hyun Cho

Nature Chemistry http://dx.doi.org/10.1038/nchem.1233 (2012); published online 10 January 2012; corrected after print 18 January 2012.

In the version of this Article originally published, it erroneously stated the authors had no competing financial interests. This information has now been corrected in the HTML and PDF versions of the Article. 